\title{
USO DEL MODELO DE BIGELOW PARA LA DETERMINACIÓN DEL VALOR D DE Listeria monocytogenes
}

\section{USE OF BIGELOW MODEL FOR THE DETERMINATION OF THE D VALUE OF Listeria monocytogenes}

\author{
Blanca Lucía Botina ${ }^{1}$ \\ María Consuelo Vanegas ${ }^{2}$ \\ Adriana $\mathrm{Coral}^{3}$
}

\section{RESUMEN}

La estimación de los parámetros de inactivación térmica de los microorganismos permite designar un tratamiento de control adecuado dentro de los procesos de transformación en la producción de alimentos. Es por esto que en el presente estudio, se determinaron estos parámetros, a partir de la constante de velocidad de destrucción térmica (k), utilizando el modelo de inactivación de Bigelow en diferentes cepas de Listeria monocytogenes. Este microorganismo ha sido aislado de alimentos y es el causante de enfermedades, como listeriosis, de la cual se han reportado altas tasas de mortalidad. Seis cepas de L. monocytogenes sembradas individualmente fueron expuestas a temperaturas de $55^{\circ}, 60^{\circ}, 65^{\circ}, 70^{\circ}$ y $72^{\circ} \mathrm{C}$ durante tiempos definidos para cada una de ellas, de igual forma, se sometió a reto térmico la mezcla de las seis cepas utilizadas. El recuento de las células viables, se realizó por medio de la técnica de recuento en placa. Los valores $\mathrm{D}$ obtenidos a $55^{\circ}$, $60^{\circ}, 65^{\circ}, 70^{\circ}$ y $72^{\circ} \mathrm{C}$ oscilaron entre 5-12,79; 2,64-3,86;

${ }^{1}$ Microbióloga. Laboratorio de Ecología Microbiana y de Alimentos (LEMA). Departamento de Ciencias Biológicas. Facultad de Ciencias. Universidad de los Andes. Cr $1 \mathrm{~N}^{\circ} 18$ A - 10. Edificio J, Laboratorio 209. b-botina@uniandes. edu.co

${ }^{2}$ Microbióloga. M.Sc. en Microbiología. Directora LEMA. mvanegas@uniandes.edu.co

${ }^{3}$ Microbióloga. Directora Gestión de Calidad Carulla Vivero S.A. Cr 68D No21-35. acoral@carullavivero.com
1,51-2,39; 0,91-1,75 y 0,66-1,03min. respectivamente. Se observó que los valores $\mathrm{D}$ más altos en todas las temperaturas fueron los presentados por las cepas de alimentos y de ambientes de plantas procesadoras, en comparación con aquellos pertenecientes a la cepa referencia. Debido a que no se ha determinado la razón por la cual estas cepas presentaron alta resistencia térmica, se recomienda seguir investigando este microorganismo en matrices similares o en alimentos y de esta manera obtener datos que puedan ser utilizados en modelos matemáticos de inactivación.

Palabras clave: Listeria monocytogenes, modelo de Bigelow, valor D.

\section{SUMMARY}

The estimate of thermal inactivation parameters of microorganisms allows to design a pathogen control treatment, suitable for the transformation processes in food production. For this reason, in this study these parameters were determined, using the speed constant of thermal destruction ( $\mathrm{k}$ ) and the Bigelow inactivation model in different Listeria monocytogenes strains; this microorganism has been isolated from foods and is the cause of diseases like listeriosis of which high rates of mortality have been reported. Six L. monocytogenes strains were spread individually in brain heart infusion broth, exposed at $55^{\circ}, 60^{\circ}, 65^{\circ}, 70^{\circ}$ and $72^{\circ} \mathrm{C}$ for different times for each temperature, at the same time a mixture of the six strains, were exposed to thermal exposure. The count of viable cells was done using the standard plate 
count technique. The D values obtained at $55^{\circ}, 60^{\circ}, 65^{\circ}$, $70^{\circ}$ and $72^{\circ} \mathrm{C}$ fluctuated between $5-12,79 ; 2,64-3,86$; 1,51-2,39; 0,91-1,75 and 0,66-1,03 min, respectively. The highest $\mathrm{D}$ values at all temperatures were obtained from food strains and environment food processing industries strains, in comparison with the reference strain values. Since the reason why these stocks presented thermal resistance has not been determined, it is recommended to continue investigating this microorganism in similar matrices or foods and to collect data that can be used in mathematical models of inactivation.

Key words: Listeria monocytogenes, Bigelow model, $\mathrm{D}$ value.

\section{INTRODUCCIÓN}

Listeria monocytogenes es una bacteria emergente, transmitida por alimentos que causa listeriosis, abortos, mortinatos y otras enfermedades en humanos y animales (Swaminathan \& Gerner, 2007). Este patógeno tiene la capacidad de sobrevivir en condiciones ambientales adversas, como temperaturas de refrigeración y es una de las bacterias más resistentes al calor en comparación con otros patógenos no esporulados (Novak et al. 2003); además es un problema importante para la industria, debido a que tiene la habilidad de generar biopelículas sobre superficies (ICMSF, 2002).

Durante los últimos años, se ha considerado a $L$. monocytogenes como uno de los microorganismos patógenos de mayor importancia en salud pública a nivel mundial, debido a los numerosos brotes reportados. Informes epidemiológicos han indicado, desde 1981 hasta el 2003, una incidencia de listeriosis entre el 0,1 y 11,3 por cada 1,000,000 de habitantes en diferentes países y una alta tasa de mortalidad, la cual ha oscilado entre el 15 y el $40 \%$ debido al consumo de alimentos contaminados como ensaladas de col, leche pasteurizada, queso, paté, lengua de cerdo, perros calientes, carnes listas para el consumo y mantequilla reportados en países, como Canada, Estados Unidos, Dinamarca, Francia e Inglaterra (Swaminathan $\mathcal{E}$ Gerner, 2007). En Colombia existen pocos reportes de esta enfermedad, ya que la mayoría de los casos clínicos no se diagnostican; sin embargo, en un estudio retrospectivo de casos de listeriosis desde 1994 hasta 1998 realizado en Cali, Colombia, se encontró un total de 19 casos y una tasa de mortalidad del $26 \%(5 / 19)$ (Torres et al. 2004).

L. monocytogenes no es un microorganismo de notificación obligatoria en Colombia, por esta razón existen pocos reportes que indiquen la presencia de este patógeno en una variedad de productos. Torres et al. (2004) mencionan, entre otros autores, a Carrascal quién reportó en 1994 y en 1996 una incidencia de 62/168 y 45/150, respectivamente, en leche cruda; igualmente citan a Muñoz et al., autores que encontraron una incidencia de 94/120 en queso fresco, mientras que Carrascal \& Mosos mostraron una incidencia de 8/24 en carne fresca y 57/172 en queso fresco. La Secretaria Distrital de Salud encontró una incidendencia de 29/633 en derivados lácteos y 1/295 en muestras de jamón de cerdo, durante el 2000-2002. En los años 2003-2006, se realizaron varios estudios, los cuales mostraron una incidencia de $12,85 \%$ en cárnicos cocidos, 1/34 en leche cruda, 45/152 en queso fresco y $1 / 8$ en queso blanco (Vanegas et al. 2006; Torres et al. 2004). Esto demuestra que, el control de $L$. monocytogenes en los productos anteriormente mencionados, aún es un desafío para la industria colombiana.

Como herramienta de control en la industria de alimentos, se han implementado los tratamientos térmicos con el objetivo de destruir poblaciones de microorganismos presentes en los alimentos y así garantizar la inocuidad y la vida útil de los mismos (Asselt $\varepsilon$ Zwietering, 2006; Wilson E Droby, 2001). Teniendo en cuenta las ventajas de este tratamiento varias investigaciones se han enfocado en la evaluación de la resistencia térmica de $L$. monocytogenes sobre matrices alimentarias en productos lácteos y cárnicos, proporcionando información interesante, como el indicar que las cepas presentes en productos curados o con aditivos logran una resistencia térmica, debido a la presencia de sales, de grasa adicionada y de texturizantes, como la proteína de soya, afectando la cinética de penetración del calor a los alimentos (Soyutemüz E Etünkaya, 2005; Passos E Kuaye, 2002).

La estimación de los parámetros de inactivación, como valor $\mathrm{D}$ o tiempo de reducción decimal, que se define como el tiempo en minutos requerido para destruir el $90 \%$ de la población inicial y el valor $Z$, que corresponde al incremento de temperatura necesario para reducir diez veces el valor D (Mossel $\&$ Moreno, 2003; Doyle 
et al. 2001), permite designar un tratamiento térmico adecuado basado en la sensibilidad o la resistencia del microorganismo. Para el cálculo de estos parámetros, se han desarrollado modelos matemáticos que describen la cinética microbiana de crecimiento o de inactivación de los microorganismos sometidos bajo ciertas condiciones o tratamientos que generan información de manera rápida y precisa, permitiendo la optimización y el diseño de un proceso térmico industrial (McMeekin et al. 1997; Valdramidis et al. 2006). Dentro de los modelos más antiguos que describen la inactivación térmica, se encuentra el modelo de Bigelow (Ecuación 1), el cual ha sido utilizado para obtener información de los parámetros térmicos necesarios para el control de patógenos en industrias de enlatados (Geeraerd et al. 2005).

\section{Ecuación 1:}

$$
\begin{aligned}
\log _{10}(\mathrm{~N})=\log _{10}\left(\mathrm{~N}_{(0)}\right) & -\frac{\mathrm{kt}}{\mathrm{Ln} 10} \\
& =\log _{10}\left(\mathrm{~N}_{(0)}\right)-\frac{\mathrm{t}}{\mathrm{D}}
\end{aligned}
$$

En esta ecuación, $N$ representa la densidad de población microbiana; $\mathrm{N}_{(0)}$ la densidad de población inicial; $\mathrm{k}$ la constante de velocidad de destrucción térmica (tiempo$\left.{ }^{1}\right)$ a temperatura constante y $\mathrm{t}$ el tiempo de procesado térmico. El modelo asume que todas las células de la población inicial tienen igual sensibilidad al calor y que su muerte depende del calor que reciba cada uno de los microorganismos (Geeraerd et al. 2005).
Los tratamientos térmicos son los métodos de control más utilizados en la industria de alimentos, por lo tanto, con el fin de proveer información útil sobre la termorresistencia de cepas nativas de L. monocytogenes, se determinó el valor $\mathrm{D}$ de cepas aisladas de alimentos y de ambientes de empresas productoras de alimentos de nuestro medio a diferentes temperaturas aplicando uno de los modelos predictivos de inactivación.

\section{MATERIALES Y MÉTODOS}

Cepas: Seis cepas de $L$. monocytogenes, aisladas de diferentes fuentes, fueron analizadas en el presente estudio (Tabla 1). Todas las cepas fueron confirmadas por PCR en el Laboratorio de Ecología Microbiana de Alimentos (LEMA) de la Universidad de los Andes (Medrano E Vanegas, 2006). Las cepas, se mantuvieron en agar Infusión Cerebro Corazón (BHI) (Sharlou Chemie S.A.), con $3 \%$ de sangre de cordero a $4^{\circ} \mathrm{C}$, durante la investigación.

Estandarización del inoculo: La cepa LMO 01 fue cultivada en caldo $\mathrm{BHI}$ a $37^{\circ} \mathrm{C}$ por $24 \mathrm{~h}$. Se realizaron diluciones seriadas $\left(10^{-1}-10^{-9}\right)$ en agua peptonada $0,1 \%$ y sembradas en superficie sobre agar Standar Plate Count (SPC) (Sharlou Chemie S.A.) a $37^{\circ} \mathrm{C}$ por $24 \mathrm{~h}$ para el conteo de células viables; de esta manera, se determinó la concentración de la población inicial. El procedimiento se realizó tres veces antes de efectuar el tratamiento térmico, el cual se utilizó como referencia. Adicionalmente, el crecimiento del cultivo

Tabla 1. Cepas de L. monocytogenes usadas en esta investigación.

\begin{tabular}{|c|c|c|}
\hline Cepa número & $\begin{array}{c}\text { Código de cepa de } \\
\text { L. monocytogenes }\end{array}$ & Origen \\
\hline 1 & LMO 01 & $\begin{array}{c}\text { ATCC 7644 } \\
\text { American Type Culture Colection }\end{array}$ \\
\hline 2 & LMO 86 serotipo $4 \mathrm{a}$ & Sifón de planta de Cárnicos \\
\hline 3 & LMO 09 serotipo $4 \mathrm{~b}$ & Queso \\
\hline 4 & LMO133 serotipo $4 \mathrm{~b}$ & Longaniza \\
\hline 5 & LMO 79 serotipo $4 \mathrm{~b}$ & Leche \\
\hline 6 & LMO 52 serotipo $4 \mathrm{a}$ & Jamón \\
\hline
\end{tabular}


fue monitoreado a 600nm con un espectrofotómetro, después de las $24 \mathrm{~h}$ de cultivar la cepa en caldo BHI.

Tratamiento térmico: La exposición térmica se realizó en baño maría (Selecta Persistem), donde se somete cada una de las cepas y una mezcla de estas a temperaturas de $55^{\circ}, 60^{\circ}, 65^{\circ}, 70^{\circ}$ y $72^{\circ} \mathrm{C}$; la temperatura interna de los tubos fue controlada con termómetro. Después del tratamiento térmico, los tubos fueron retirados del baño maría a diferentes intervalos de tiempo definidos (Tabla 2). El recuento de $U F C / m L$ de células viables fue determinado en agar SPC a $37^{\circ} \mathrm{C}$ por 24 horas.

Tabla 2. Intervalos de tiempo en los cuales los tubos del baño maría fueron retirados, según el reto térmico.

\begin{tabular}{|c|c|}
\hline Temperatura $\left({ }^{\circ} \mathrm{C}\right)$ & Tiempo (s) \\
\hline 55 & 180 \\
\hline 60 & 90 \\
\hline 65 & 60 \\
\hline 70 & 45 \\
\hline 72 & 25 \\
\hline
\end{tabular}

Cálculo del valor D: La resistencia térmica de las cepas fue estimada por la determinación de los valores D para cada una de las temperaturas evaluadas. Este valor se obtuvo a partir del modelo lineal (Ecuación 1), utilizando el negativo de la inversa multiplicativa de la pendiente k/Ln 10, cuyo valor se logró utilizando el programa "Inactivation Model Fitting Tool, Katholieke Universiteit Leuven, Bélgica, para cada uno de los retos térmicos.

Los experimentos, se realizaron por duplicado, obteniendo un valor $\mathrm{D}$ para cada una de las réplicas, donde el valor $D_{1}$ es el valor $D$ de la primera réplica y $\mathrm{D}_{2}$ el valor $\mathrm{D}$ de la segunda. La correlación de los datos respecto a las pendientes de cada réplica, se analizó con el software Statistix 8 (Versión 8. Analytical Software, Estados Unidos, 2003), para denominar el valor D común, que se obtuvo al utilizar el promedio de los datos de las dos réplicas.

\section{RESULTADOS Y DISCUSIÓN}

Los recuentos obtenidos en las tres réplicas realizadas para la estandarización del inoculo con la cepa de $L$. monocytogenes LMO 01 ATCC 7644 fueron de $1 \times 10^{8} \mathrm{y}$ $5 \times 10^{8} \mathrm{UFC} / \mathrm{ml}$ y una densidad óptica $\mathrm{OD}_{600 \mathrm{~nm}}$ entre 0,505 $-0,685$, indicando una población final constante de la cepa a $37^{\circ} \mathrm{C}$ durante $24 \mathrm{~h}$. Con base en esta información, se activaron las otras cepas bajo las mismas condiciones, las cuales presentaban crecimiento típico en el agar sangre, medio en el cual se mantuvieron antes de iniciar el reto térmico. Los recuentos iniciales de los inóculos estandarizados en caldo $\mathrm{BHI}$, antes de someterlos al reto térmico, oscilaron entre $1 \times 10^{8}$ y $2,9 \times 10^{9} \mathrm{UFC} / \mathrm{ml}$ (Tabla 3), valores que se tuvieron en cuenta para iniciar el tratamiento térmico.

La tabla 3 muestra los valores D para las dos réplicas. La comparación de las pendientes obtenidas mediante la regresión lineal de los datos de las dos réplicas, para todos los retos térmicos, no presentó diferencias significativas ( $p>0,05$ ); por lo tanto, para el análisis de estos datos, se tuvo en cuenta el valor común de resistencia al calor ( $D$ común) en todos los retos realizados para cada cepa sometida a temperaturas de $55,60,65,70$ y $72^{\circ} \mathrm{C}$.

Dentro de los datos reportados en la literatura, se encuentran diferentes valores de D para cepas de $L$. monocytogenes sometidas bajo una temperatura de estrés determinada, por tal razón, no es posible comparar directamente los resultados a cierta temperatura de una investigación con los de otros estudios, debido a que existen muchos factores, como el uso de diferentes 
Tabla 3. Resultados de resistencia al calor de seis cepas de Listeria monocytogenes y la mezcla de las mismas, obtenidas después de la incubación a $37^{\circ} \mathrm{C}$ por 24 horas.

\begin{tabular}{|c|c|c|c|c|c|c|c|c|c|}
\hline \multirow[b]{2}{*}{$\begin{array}{c}\text { Tempera } \\
\text { tura }\end{array}$} & \multirow[b]{2}{*}{ Cepa } & \multicolumn{2}{|c|}{ Recuento inicial } & \multicolumn{2}{|c|}{ Primera réplica } & \multicolumn{2}{|c|}{ Segunda réplica } & \multicolumn{2}{|c|}{ Común } \\
\hline & & Réplica 1 & Réplica 2 & K & $\begin{array}{c}\text { D1 } \\
\text { minutos }\end{array}$ & K & $\begin{array}{c}\mathrm{D} 2 \\
\text { minutos }\end{array}$ & K & $\begin{array}{l}\text { D común } \\
\text { minutos }\end{array}$ \\
\hline \multirow{7}{*}{55} & 1 & $2,00 \mathrm{E}+09$ & $1,50 \mathrm{E}+09$ & 0,46 & 5,00 & 0,45 & 5,11 & 0,46 & 5 \\
\hline & 2 & $1,70 \mathrm{E}+09$ & $1,40 \mathrm{E}+09$ & 0,46 & 5 & 0,39 & 5,9 & 0,43 & 5,35 \\
\hline & 3 & $1,90 \mathrm{E}+09$ & $1,30 E+09$ & 0,24 & 9,59 & 0,20 & 11,51 & 0,22 & 10,46 \\
\hline & 4 & $1,80 \mathrm{E}+09$ & $1,60 \mathrm{E}+09$ & 0,23 & 10,01 & 0,31 & 7,42 & 0,26 & 8,85 \\
\hline & 5 & $2,70 \mathrm{E}+09$ & $2,90 \mathrm{E}+09$ & 0,54 & 4,26 & 0,37 & 6,22 & 0,45 & 5,11 \\
\hline & 6 & $1,50 \mathrm{E}+09$ & $1,60 \mathrm{E}+09$ & 0,33 & 6,97 & 0,38 & 6,05 & 0,35 & 6,57 \\
\hline & MEZCLA & $1,90 \mathrm{E}+09$ & $2,10 E+09$ & 0,20 & 11,51 & 0,15 & 15,35 & 0,18 & 12,79 \\
\hline \multirow{7}{*}{60} & 1 & $1,20 \mathrm{E}+09$ & $1,30 \mathrm{E}+09$ & 0,82 & 2,8 & 0,93 & 2,47 & 0,87 & 2,64 \\
\hline & 2 & $1,50 \mathrm{E}+09$ & $1,30 \mathrm{E}+09$ & 0,73 & 3,17 & 0,50 & 4,06 & 0,60 & 3,83 \\
\hline & 3 & $1,00 E+09$ & $2,50 \mathrm{E}+09$ & 0,96 & 2,39 & 0,69 & 3,33 & 0,82 & 2,8 \\
\hline & 4 & $1,20 \mathrm{E}+09$ & $1,30 \mathrm{E}+09$ & 0,56 & 4,11 & 0,67 & 3,43 & 0,62 & 3,71 \\
\hline & 5 & $2,40 \mathrm{E}+09$ & $2,70 \mathrm{E}+09$ & 0,83 & 2,77 & 0,62 & 3,71 & 0,73 & 3,15 \\
\hline & 6 & $1,40 E+09$ & $1,70 \mathrm{E}+09$ & 0,73 & 3,15 & 0,76 & 3,02 & 0,75 & 3,07 \\
\hline & MEZCLA & $1,70 \mathrm{E}+09$ & $1,60 \mathrm{E}+09$ & 0,72 & 3,19 & 0,86 & 2,67 & 0,79 & 2,91 \\
\hline \multirow{7}{*}{65} & 1 & $1,40 \mathrm{E}+09$ & $1,60 \mathrm{E}+09$ & 1,49 & 1,54 & 1,56 & 1,47 & 1,52 & 1,51 \\
\hline & 2 & $1,90 \mathrm{E}+09$ & $1,40 \mathrm{E}+09$ & 1,10 & 2,09 & 1,34 & 1,71 & 1,22 & 1,88 \\
\hline & 3 & $1,00 E+09$ & $1,30 E+09$ & 1,36 & 1,69 & 1,53 & 1,50 & 1,45 & 1,58 \\
\hline & 4 & $1,80 \mathrm{E}+09$ & $1,40 \mathrm{E}+09$ & 1,39 & 1,65 & 0,92 & 2,50 & 1,16 & 1,98 \\
\hline & 5 & $1,60 \mathrm{E}+09$ & $1,70 \mathrm{E}+09$ & 1,15 & 2,00 & 1,25 & 1,84 & 1,2 & 1,91 \\
\hline & 6 & $1,10 E+09$ & $1,20 \mathrm{E}+09$ & 0,98 & 2,34 & 0,98 & 2,34 & 0,96 & 2,39 \\
\hline & MEZCLA & $2,00 \mathrm{E}+09$ & $1,70 \mathrm{E}+09$ & 1,36 & 1,69 & 2,18 & 1,05 & 1,17 & 1,96 \\
\hline \multirow{7}{*}{70} & 1 & $1,00 \mathrm{E}+09$ & $1,80 \mathrm{E}+09$ & 2,64 & 0,9 & 2,5 & 0,92 & 2,52 & 0,91 \\
\hline & 2 & $2,40 \mathrm{E}+09$ & $1,90 \mathrm{E}+09$ & 2,12 & 1,09 & 1,59 & 1,44 & 1,87 & 1,23 \\
\hline & 3 & $1,80 \mathrm{E}+09$ & $2,60 \mathrm{E}+09$ & 1,97 & 1,16 & 1,70 & 1,35 & 2 & 1,15 \\
\hline & 4 & $1,30 \mathrm{E}+09$ & $1,10 \mathrm{E}+09$ & 1,69 & 1,36 & 1,93 & 1,19 & 1,81 & 1,27 \\
\hline & 5 & $1,80 \mathrm{E}+09$ & $1,50 \mathrm{E}+09$ & 1,80 & 1,27 & 1,61 & 1,43 & 1,76 & 1,3 \\
\hline & 6 & $1,30 \mathrm{E}+09$ & $1,70 \mathrm{E}+09$ & 2,00 & 1,15 & 2,37 & 0,97 & 2,17 & 1,06 \\
\hline & MEZCLA & $1,50 \mathrm{E}+09$ & $1,80 \mathrm{E}+09$ & 1,54 & 1,49 & 1,01 & 2,27 & 1,31 & 1,75 \\
\hline \multirow{7}{*}{72} & 1 & $1,10 \mathrm{E}+09$ & $1,40 \mathrm{E}+09$ & 3,04 & 0,75 & 4,07 & 0,56 & 3,44 & 0,66 \\
\hline & 2 & $1,60 \mathrm{E}+09$ & $1,20 \mathrm{E}+09$ & 2,06 & 1,11 & 2,37 & 0,97 & 2,23 & 1,03 \\
\hline & 3 & $1,50 \mathrm{E}+09$ & $1,10 \mathrm{E}+09$ & 2,36 & 0,97 & 2,26 & 1,01 & 0,28 & 1 \\
\hline & 4 & 1,70E+09 & $1,90 \mathrm{E}+09$ & 2,35 & 0,97 & 2,93 & 0,78 & 2,66 & 0,86 \\
\hline & 5 & $2,90 \mathrm{E}+09$ & $2,50 \mathrm{E}+09$ & 2,67 & 0,86 & 2,92 & 0,78 & 2,82 & 0,81 \\
\hline & 6 & $2,10 \mathrm{E}+09$ & $1,90 \mathrm{E}+09$ & 3,35 & 0,68 & 2,74 & 0,84 & 3,04 & 0,75 \\
\hline & MEZCLA & $1,30 E+09$ & $1,10 E+09$ & 2,39 & 0,96 & 2,42 & 0,95 & 2,4 & 0,95 \\
\hline
\end{tabular}

k $\quad$ Constante de velocidad de destrucción térmica

D1 Valor de resistencia al calor de la primera réplica.

D2 Valor de resistencia al calor de la segunda réplica.

$\mathrm{D}$ (común) Valor de resistencia al calor teniendo en cuenta los valores de las réplicas 1 y 2 
medios de cultivo, procedimientos usados para la numeración de células sobrevivientes y condiciones experimentales que difieren unas de otras (Doyle et al. 2001). Es por esto que los datos obtenidos en este estudio, se analizaron con base a los datos de otras investigaciones bajo condiciones experimentales similares, más no idénticas.

El valor D determinado por Pachett et al. (1996) para la cepa de L. monocytogenes NCTC 7973 cultivada en medio PB en quimiostato y sometida a $55^{\circ} \mathrm{C}$ fue de 6,3min (Doyle et al. 2001). Este valor es muy cercano al obtenido en el presente estudio para la cepa LMO 52 aislada de jamón y sometida a la misma temperatura, el cual fue de $6,57 \mathrm{~min}$. El valor $\mathrm{D}_{60^{\circ} \mathrm{C}}$ obtenido para la cepa LMO 86 aislada de sifón fue de 3,83min, el cual es muy cercano al reportado para la cepa Lm5S con un valor $\mathrm{D}$ de $4,1 \mathrm{~min}$, la cual fue incubada en TSB con piruvato a $37^{\circ} \mathrm{C}$, por tres días (Doyle et al. 2001). La cepa SLU 196 serovar 3b reporta un valor $\mathrm{D}_{64^{\circ} \mathrm{C}}$ de 0,68min (Sörqvist, 1994), dato menor a los valores D obtenidos a $65^{\circ} \mathrm{C}$ para todas las cepas en este estudio analizadas, los cuales oscilan entre $1,51 \mathrm{~min}$, como valor mínimo correspondiente a la cepa LMO 01 y valor máximo 2,39min, determinado para la cepa LMO 52 aislada de jamón.

Hasta el momento no se ha encontrado información disponible en donde se haya utilizado un medio de cultivo como matriz sintética para las temperaturas de 70 y $72^{\circ} \mathrm{C}$; sin embargo, estas temperaturas han sido evaluadas en matrices de alimentos, como carne de res, en donde se han encontrado valores $D_{70^{\circ} \mathrm{C}}$ de $0,47 \mathrm{~min}$ para la cepa CRA 433. Para la mezcla de tres cepas de L. monocytogenes referenciadas como 101M, F6854 y CLIP23485 inoculadas en salchichas, se han reportado valores $D_{77^{\circ} \mathrm{C}}$ de $0,84 \mathrm{~min}$ y a $88^{\circ} \mathrm{C}$ valores $\mathrm{D}$ de 0,37 min (Doyle et al. 2001). La cepa F5069 inoculada en leche entera presentó un valor $\mathrm{D}_{71.7^{\circ} \mathrm{C}}$ de $0.077 \mathrm{~min}$. Estos valores reportados para el rango de temperaturas entre 70 y $77^{\circ} \mathrm{C}$ son muy bajos en comparación con los obtenidos en este estudio, donde los valores a $70^{\circ} \mathrm{C}$ y $72^{\circ} \mathrm{C}$ se encuentran entre $0,91-1,75 \mathrm{~min}$ y $0,66-0,95 \mathrm{~min}$, respectivamente, para la cepa LMO 01 ATCC 7644 y para la mezcla de todas las cepas en este estudio evaluadas.

Los resultados de este estudio indican que la mayoría de los valores $\mathrm{D}$ calculados se encuentran en el rango de los valores más altos dentro de los reportados en otras investigaciones realizadas bajo condiciones similares de laboratorio (Doyle et al. 2001, Sörqvist, 1994), por lo cual, se aconsejaría trabajar una metodología que permita obtener datos más precisos, incluyendo tres réplicas para poder hacer uso de modelos de inactivación que presenten un mejor ajuste de los datos y, de esta manera, obtener para todos los casos valores de $\mathrm{R}^{2}$ mayores y disminuir el error cuadrático medio.

Aunque no se conoce el origen de las cepas reportadas en la literatura con las cuales se relacionaron los resultados de esta investigación, se conoce que dentro de los factores que afectan la resistencia al calor de las cepas se encuentra el origen de las mismas (Sörqvist, 1994; Doyle et al. 2001; Asselt E Zwietering, 2006). Golden et al. (1998) evaluaron el valor $D_{56^{\circ} \mathrm{C}}$ de cepas de $L$. monocytogenes aisladas de diferentes fuentes y analizadas bajo las mismas condiciones; además, fueron aisladas de una muestra de repollo implicado en un brote, de un queso brie y de dos muestras humanas, una obtenida después de un brote causado por leche contaminada y la otra recuperada en una época diferente, con valores $D_{56^{\circ} \mathrm{C}}$ de $10,4,16,7,4$ y 5,7min, respectivamente. Así mismo, se observó que los resultados obtenidos no eran similares entre sí, a pesar de ser sometidos a las mismas condiciones (Doyle et al 2001). El mismo comportamiento, se observó al comparar los valores de las cepas trabajadas en la investigación de cepas nativas colombianas, las cuales provenían de plantas procesadoras de cárnicos, queso, longaniza, leche y jamón para cada una de las temperaturas aquí evaluadas. Igualmente, los resultados señalan que ninguno de los valores obtenidos para cada una de las temperaturas fue similar, a pesar de que las cepas se encontraban bajo las mismas condiciones (Figura 1).

La resistencia al calor para las cepas de $L$. monocytogenes según los resultados del valor $\mathrm{D}$ obtenido es inversamente proporcional a la temperatura a la que fueron sometidas. Las curvas de inactivación térmica a, b, c y d (Figura 2) y a, b y c (Figura 3) describen la destrucción de las diferentes cepas de bajo las diferentes temperaturas. Éstas exhiben una mayor pendiente de declinación lineal en la población sometida a $72^{\circ} \mathrm{C}$, razón por la cual se obtuvo un valor D menor en todas las cepas, en comparación con las otras temperaturas. Adicionalmente, todas las gráficas que representan la inactivación a $55^{\circ} \mathrm{C}$ muestran una menor pendiente de declinación bajo esta temperatura, 


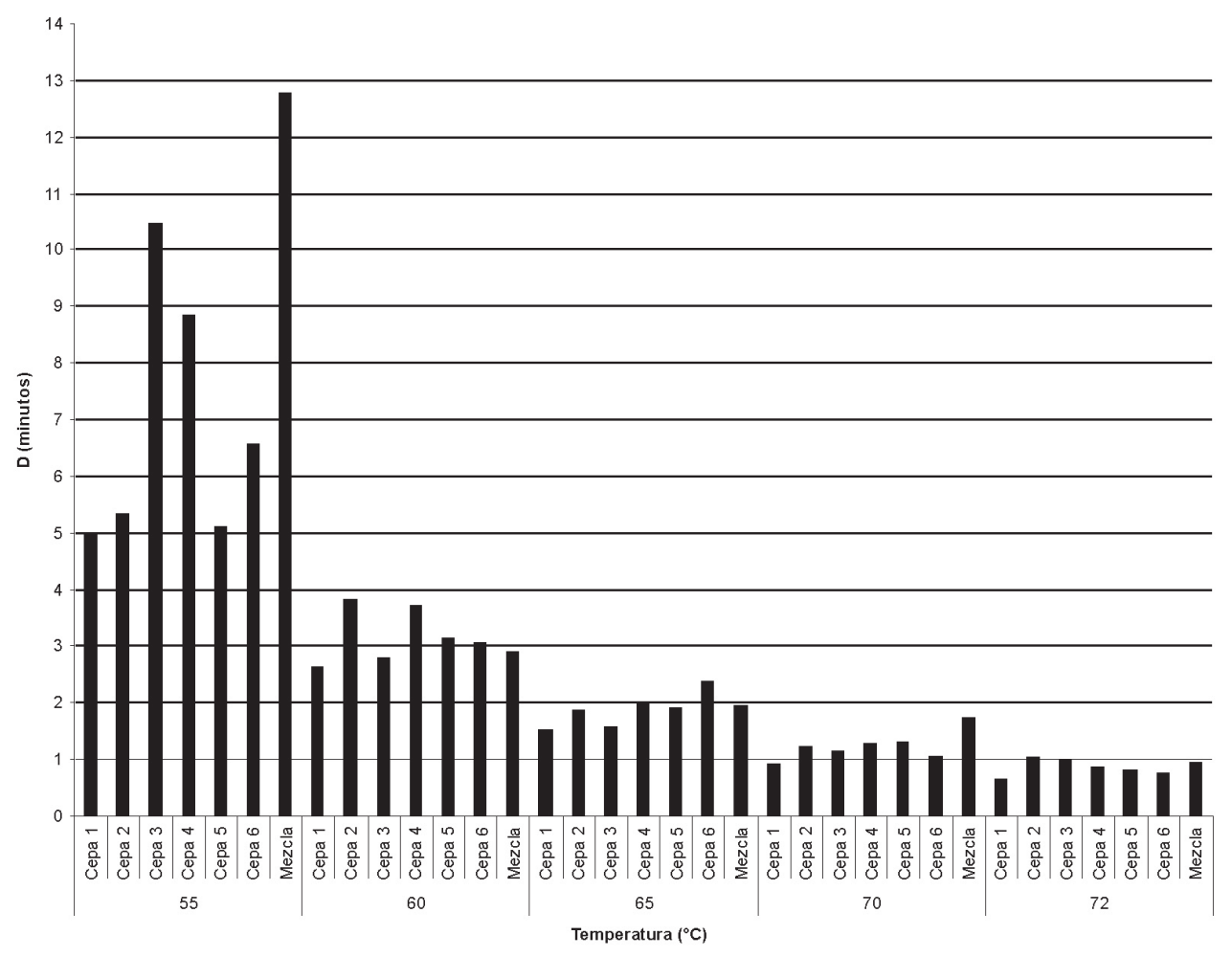

Figura 1. Valores $\mathrm{D}(\mathrm{min})$ de las diferentes cepas de L. monocytogenes bajo diferentes tratamientos de temperatura.

presentando de esta manera valores D más altos en comparación con las otras temperaturas.

Al comparar los valores $\mathrm{D}$ a diferentes temperaturas de estrés para las cepas LMO 86, LMO 09, LMO 133, LMO 79 y LMO 52, sugiere que las cepas de diferentes fuentes, muestran una mayor tolerancia a las temperaturas evaluadas en comparación con la cepa ATCC 7644. La cepa con mayor resistencia térmica del grupo de cepas estudiadas, no se pudo determinar debido a la gran variación de los valores $\mathrm{D}$ calculados en todas las temperaturas evaluadas (Tabla 3).

Estudios anteriores (Sörqvist, 1994, Juneja et al. 1998, Rowan $\mathcal{E}$ Anderson 1998) registraron un incremento en la termotolerancia de $L$. monocytogenes como resultado de previas exposiciones térmicas del microorganismo durante un determinado tiempo, lo cual es atribuido a la síntesis de proteínas especiales conocidas como proteínas de choque térmico (HSPs), como respuesta fisiológica. La exposición de las cepas LMO 86, LMO 09, LMO 133, LMO 79 y LMO 52 a diferentes condiciones adversas durante el procesamiento industrial del alimento o ambiente como los tratamientos térmicos en el que se incluyen temperaturas iguales o superiores a las que se mantienen los alimentos calientes en puntos de venta $55^{\circ} \mathrm{C}$ (Mossel \& Moreno, 1985), temperaturas de cocción $70^{\circ} \mathrm{C}$ (ICMSF, 2002), tratamientos químicos, como la presencia de sales o aditivos en los alimentos o tratamientos mecánicos, pudieron favorecer el desarrollo de mecanismos de resistencia en estos microorganismos (Doyle et al. 2001).

Las pendientes de la mezcla de las cepas evaluadas a $60^{\circ}, 65^{\circ}, 70^{\circ}$ y $72^{\circ} \mathrm{C}$ no muestran diferencias altamente significativas en comparación con los valores obtenidos para las cepas evaluadas individualmente. La pendiente obtenida para la temperatura de inactivación de $55^{\circ} \mathrm{C}$ alcanzó diferencias altamente significativas con $\mathrm{p}<0.05$ en comparación con las pendientes de las cepas evaluadas individualmente y sometidas a la misma temperatura, lo cual indica, en el presente estudio, que la presencia de una variedad de cepas en una sola matriz, no influye en la resistencia térmica de las mismas, en temperaturas mayores a $60^{\circ} \mathrm{C}$. 
a)

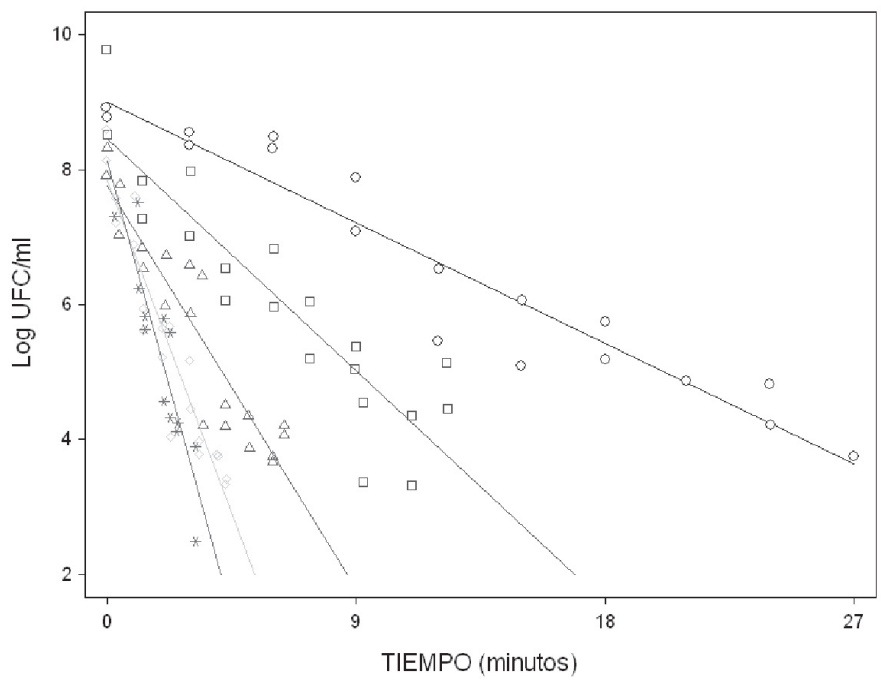

c)

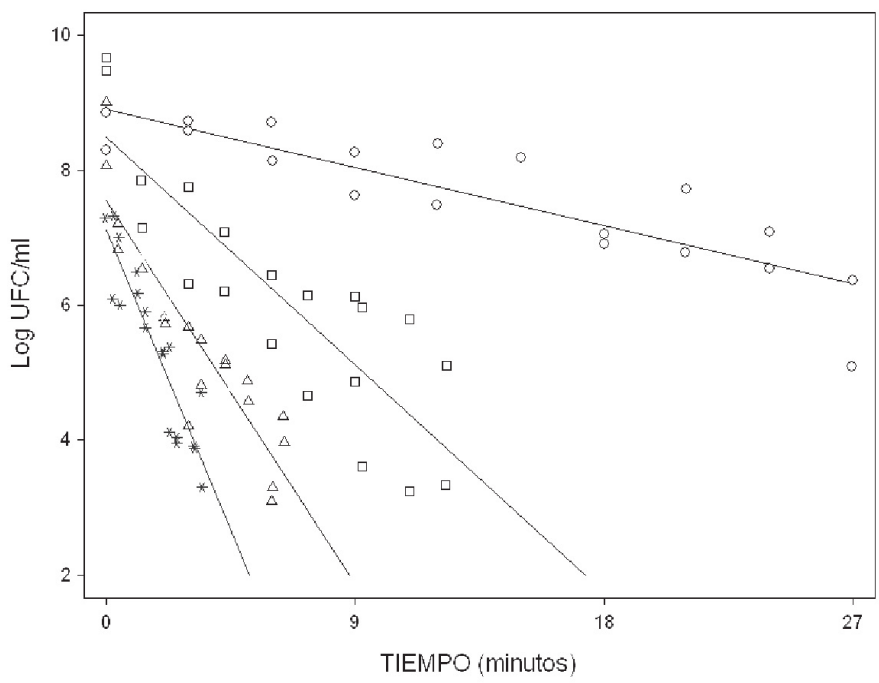

b)

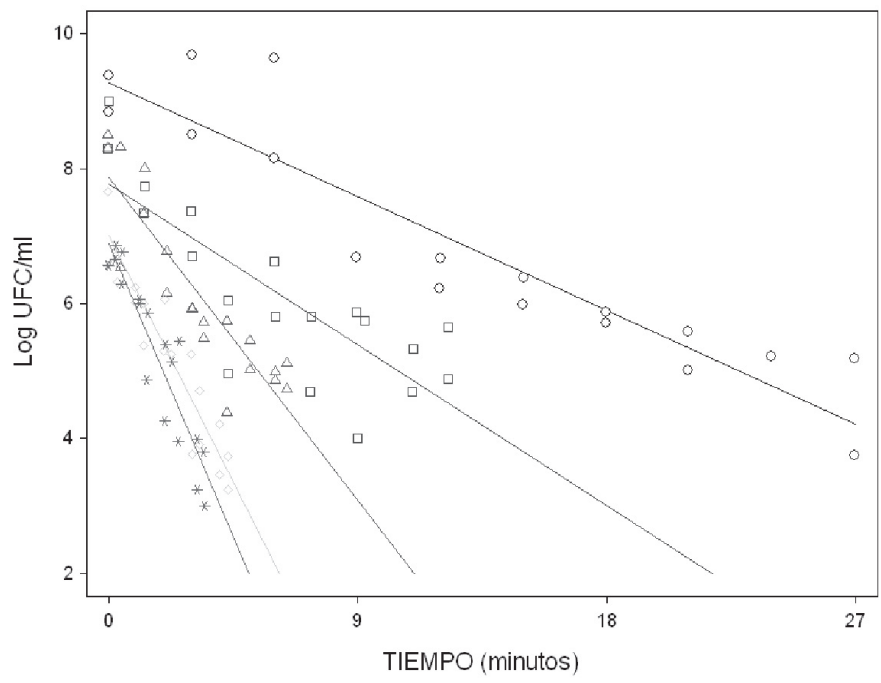

d)

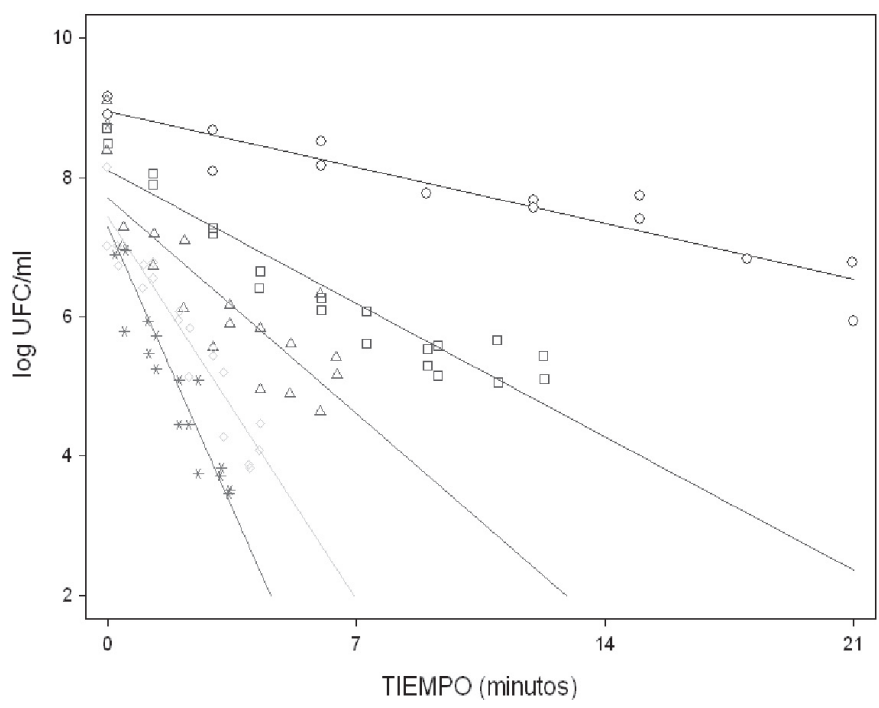

Figura 2. Curvas de inactivación térmica de Listeria monocytogenes (a) LMO 01 ATCC 7644, (b) LMO86 sifón, (c) LMO 09 queso, (d) LMO 133 longaniza, obtenidas después del reto térmico a temperaturas de $55^{\circ}(0), 60^{\circ}(\square), 65^{\circ}(\Delta), 70^{\circ}(\diamond)$ y $72^{\circ} \mathrm{C}(*)$ e incubadas a $37^{\circ} \mathrm{C}$ por 24 horas en SPC.

Los valores de resistencia térmica de las bacterias dependen del alimento o de la matriz en el que se encuentren, ya que puede contener una gran cantidad de grasa, proteína, concentración de agua o aditivos que favorecen la resistencia del microorganismo en el alimento, como lo reportan estudios realizados en queso, en leche, en carne de res, en pollo y en cerdo (Casadei et al. 1998; Doyle et al. 2001; Juneja E Belen, 1999; Mackey et al. 1990; Sörqvist, 1993). Numerosos estudios indican que $L$. monocytogenes es más resistente al calor cuando se encuentra dentro de los alimentos, debido a los altos valores de resistencia térmica en comparación con los valores obtenidos dentro de un medio de cultivo o matriz sintética de laboratorio (Casadei et al. 1998; 


\section{a)}

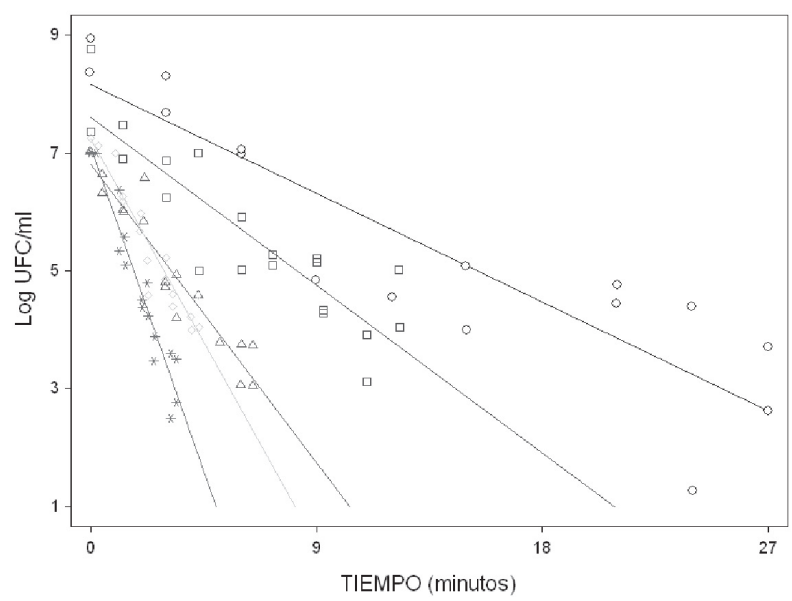

b)

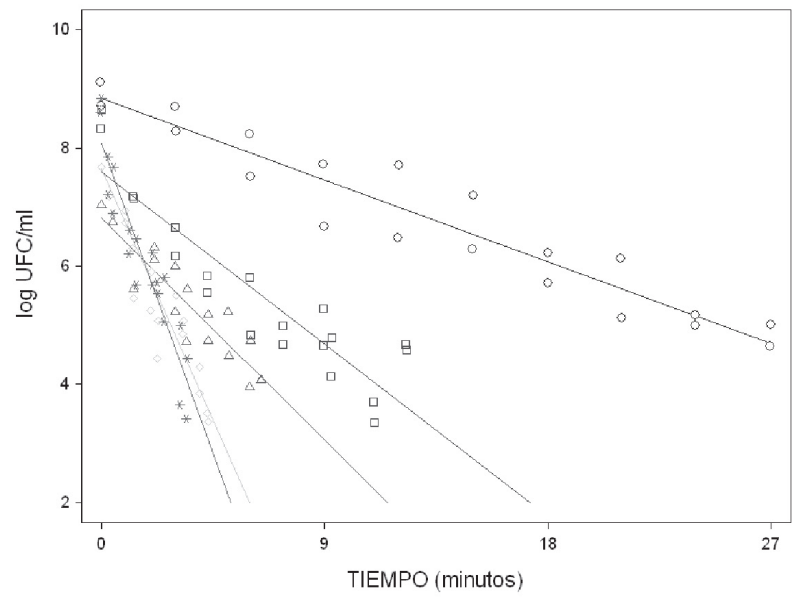

c)

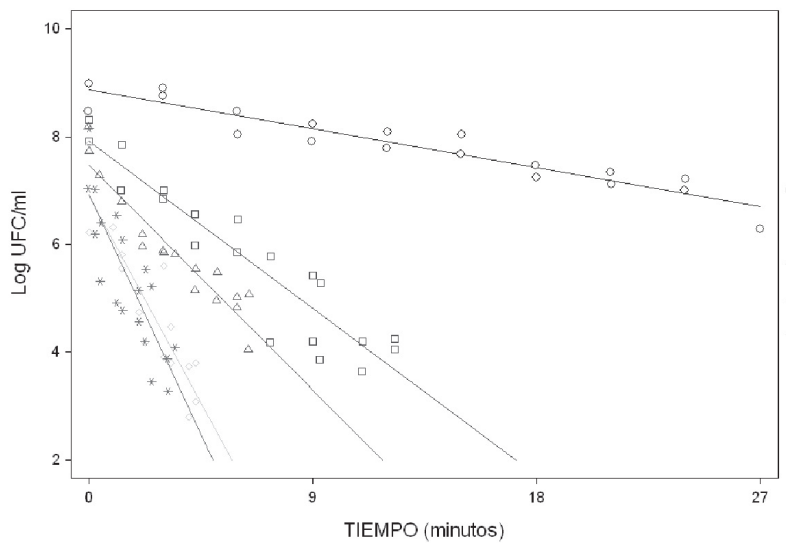

Figura 3. Curvas de inactivación térmica de Listeria monocytogenes (a) LMO 79 leche, (b) LMO 52 jamón, (c) Mezcla de cepas, obtenidas después del reto térmico a temperaturas de $55^{\circ}(0), 60^{\circ}(\square), 65^{\circ}(\Delta), 70^{\circ}(\diamond)$ y $72^{\circ} \mathrm{C}(*)$ e incubadas a $37^{\circ} \mathrm{C}$ por 24 horas en SPC. 
Doyle et al 2001). Se recomienda, entonces, encontrar los parámetros de inactivación para $L$. monocytogenes en una matriz real y, de esta manera, obtener datos que puedan ser utilizados en procesos térmicos industriales, para asociarlos al valor $F_{p}$, que corresponde al número de minutos necesario a una temperatura dada para destruir un número determinado de células viables con un valor z específico (Doyle et al. 2001).

\section{CONCLUSIONES}

Se observó que las cepas de L. monocytogenes aisladas de diferentes fuentes, como alimentos o ambiente, no se eliminaron fácilmente con temperaturas de $55^{\circ}, 60^{\circ}$, $65^{\circ}, 70^{\circ}$ y $72^{\circ}$, en comparación con la cepa referencia, teniendo en cuenta los valores D obtenidos, según el modelo de Bigelow. Aunque no se conocen las causas de la alta resistencia, se sugiere realizar estudios en los que se tenga en cuenta un mayor nuúmero de cepas de diferente serovariedad, origen, condición de crecimiento, es decir, viabilidad de la cepa en medios de cultivo y así obtener datos que se puedan interpretar por medio de modelos matemáticos lineales, como el de Bigelow o no lineales como los de Geeraerd, Mafart y Weibull, que permitan conocer información sobre la cinética de inactivación de este patógeno, con el fin de poder determinar el tratamiento térmico adecuado que garantice la inocuidad de un alimento cocido.

\section{AGRADECIMIENTOS}

Agradecemos al Comité de Investigaciones de la Facultad de Ciencias de la Universidad de Los Andes, así como también la asesoría y el apoyo brindado por el doctor Bernardo Clavijo, en relación al tema de microbiología predictiva.

\section{BIBLIOGRAFÍA}

ASSELT, E.D.; ZWIETERING, M.H. 2006. A systematic approach to determine global thermal inactivation parameters for various food pathogens. Internal J. Food Microbiol. 107:73-82

CASADEI, M.A.; ESTEVES DE M., R.; HARRISSON, S.T.; GAZE, J.E. 1998. Heat resistance of Listeria monocytogenes in dairy products as affected by the growth medium. J. Appl. Microbiol. 84:234-239.

DOYLE, M.E.; MAZZOTTA, A.S.; WANG, T.; WISEMAN, D.W.; SCOTT, V.N. 2001. Heat resistance of Listeria monocytogenes. J. Food Protection. 64 (3):410-429.

DOYLE, M.P.; BEUCHAT, L.R.; MONTVILLE, T.J. 2001. Microbiología de los alimentos: fundamentos y fornteras. Primera edición Ed. Acribia S.A. Zaragoza (España) p.527-530.

GEERAERD, A.H.; VALDRAMIDIS, V.P.; VAN IMPET, J.F. 2005. GInaFiT, a freeware tool to assess non-loglinear microbial survivor curves. Internal. J. Food Microbiol. 102:95-105.

GOLDEN, D.A.; BEUCHAT, L.R.; BRANKETT, R.E. 1998. Inactivation and injury of Listeria monocytogenes as affected by heating and freezing. Food Microbiol. 5:17-23.

ICMSF. 2002. Microorganisms in food 7 microbial testing in food safety management. Kluwer Academic Plenum Publishers. p.287-289.

JUNEJA, V.K.; BELEN, B.S. 1999. Predicitive thermal inactivation model for Listeria monocytogenes with temperature, $\mathrm{pH}, \mathrm{NaCl}$ and sodium pyrophosphate as controlling factors. J. Food Protec. 628(9): 986-993.

JUNEJA, V.K.; KLEIN, P.G.; MARMER, B.S. 1998. Heat shock and thermotolerance of Escherichia coli O157:H7 in a model beef gravy system and ground beef. J. Appl. Microbiol. 84:677-684.

MACKEY, B.M.; PRITCHET, C.; NORRIS, A. 1990. Heat resistance of Listeria: strain differences and effects of meat type and curing salts. Letters Appl. Microbiol. 10:251-255.

MCMEEKIN, T.A.; BROWN, J.; KRIST, K.; MILES, D.; NEYMEYER, K.; NICHOLS, D.S.; OLLEY, J.; PRESSER, K; RATKOWSKY, D. A.; ROSS, T.; SALTER M.; SOONTRANON, S. 1997. Quantitative microbilogy: A basis for food safety. Em. Infect. Diseases. 3(4):541-548. 
MEDRANO, M.; VANEGAS, C. 2006. Molecular Typing of Listeria monocytogenes isolates from different food processing environments and Food. Memorias. Final Program American Society for microbiology $106^{\text {th }}$. General meeting. Orlando Florida. May 21-25.

MOSSEL, D.A.A.; MORENO, B. 2003. Microbiología de

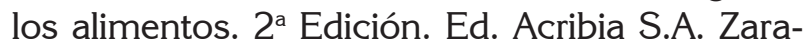
goza (España). p.526-530.

MOSSEL, D.A.A.; MORENO, B. 1985. Microbiología de los alimentos. $1^{a}$ Edición. Ed. Acribia S.A. Zaragoza (España) p.108, 119-120.

NOVAK, J.S.; SAPERS, G.M.; JUNEJA, V.K. 2003. Microbial safety of minimally processed food. $1^{\text {rst }}$ ed. CRC Press. (Boca Raton, EEUU). p.120-132.

PATCHETT, R.A.; WATSON, N.; FERNANDEZ, P.S.; KROLL, R.G. 1996. The effect of temperature and growth rate of the susceptibility of Listeria monocytogenes to environmental stress conditions. Letters. Appl. Microbiol. 22:121-124

PASSOS, M.H.; KUAYE, A.Y. 2002. Influence of formulation, cooking time and final internal temperature of beef hamburger on the destruction of Listeria monocytogenes. Food Control 13:33-40

ROWAN N.; ANDERSON J. 1998. Effects of above-optimun growth temperature and cell morphology on thermotolerance of $L$. monocytogenes cells suspended in bovine milk. Appl. Environ. Microbiol. 64(6):2065-2071.

SÖRQVIST, S. 1993. Heat resistance of Listeria monocytogenes by two recovery media used with and without cold preincubation. J. Appl. Microbiol.. 74:428-432.
SÖRQVIST, S. 1994. Heat resistance of different serovars of Listeria monocytogenes. J. Appl. Microbiol. 76:383-388.

SOYUTEMÜZ, G.E.; ETÜNKAYA, F. 2005. Thermal Resistance of Listeria monocytogenesin Üneg.l Meatballs Turk. J. Vet. Anim. Sci. 29:319-323

SWAMINATHAN, B.; GERNER, P. 2007. The epidemiology of human listeriosis. Microbes and Infection. 9:1236- 1243

TORRES, K.J.; SIERRA, S.C.; POUTOU, R.A.; VERA, H.; CARRASCAL, A.K.; MERCADO, M. 2004. Incidencia y diagnóstico de Listeria monocytogenes microorganismo zoonótico emergente en la industria de alimentos. Rev. U.D.C.A Act. \& Div. Cient. (Colombia). 7(1):27-57.

VANEGAS, M.C.; MARTÍNEZ, A.J.; BOTINA, B.L. 2006. Detección por PCR de Listeria monocytogenes en productos cárnicos distribuidos en Bogota. Rev. U.D.C.A Act. \& Div. Cient. 9(2):149-156.

VALDRAMIDIS, V.P; GEERAERD, A.H.; GAZE, J.E.; KONDJOYAN, A., BOYD, A.R.; SHAW, H.L.; VAN IMPE, J.F. 2006. Quantitative description of Listeria monocytogenes inactivation kinetics with temperature and water activity as the influencing factors; model prediction and methodological validation on dynamic data. J. Food Engineering. 76:79-88.

WILSON, C.L.; DROBY, S. 2001. Microbial food contamination. Primera edición. CRC Press. (Boca Raton, EE(IU). p.172-186.

Recibido: diciembre 13 de 2006

Aceptado: octubre 23 de 2007 